\title{
Avaliação de metais em pescado da região do Pontal da Barra, Laguna dos Patos, Pelotas-RS
}

\author{
P.J. Sanches Filho; V.K. da Fonseca \& L. Holbig \\ Grupo de Pesquisa em Contaminantes Ambientais - Instituto Federal de Educação, Ciência \\ e Tecnologia Sul-rio-grandense - Campus Pelotas
}

(Received May 18, 2012; Accept November 30, 2012)

\begin{abstract}
Resumo
Os metais pesados têm a capacidade de acumular-se em tecidos vivos ao longo da cadeia alimentar. Peixes podem se tornar a principal forma de transferir para a população esses elementos, uma vez que eles são capazes de bioacumulação e bioconcentração. Com o objetivo de investigar a bioacumulação nos peixes no Pontal da Barra-Laguna dos Patos, em Pelotas, foram escolhidas três espécies abundantes na região, Micropogonias furnieri (Corvina), Mugil platanus (Tainha) e Netuma barba (Bagre). Amostras da sua musculatura foram analisados para verificar o conteúdo de $\mathrm{Al}, \mathrm{Cd}, \mathrm{Cr}, \mathrm{Cu}, \mathrm{Fe}, \mathrm{Mn}, \mathrm{Ni}, \mathrm{Pb}$ e $\mathrm{Zn}$. Amostras em quintuplicata sofreram digestão ácida $\mathrm{HNO}_{3} / \mathrm{HCIO}_{4}$, sendo os extratos analisados por plasma indutivamente acoplado - espectrometria de emissão óptica (ICP-OES). Os níveis de cromo encontrados nas três espécies estavam acima dos estabelecidos pela legislação brasileira (Decreto-Lei 55,871-65), que determinam como um nível máximo tolerado para o cromo $0,1 \mathrm{mg} \mathrm{kg}^{-1}$. Os $82,46 \pm 4,69 \% \mathrm{mg} \mathrm{kg}^{-1}$ de zinco na espécie, Netuma barba, também excedeu o limite permitido. As concentrações de cádmio encontrada na espécie Micropogonias furnieri, $0,92 \pm 8,16 \% \mathrm{mg} \mathrm{kg}^{-1}$ ficaram próximas ao limite permitido. Os demais analitos, encontraram-se em níveis inferiores aos estabelecidos pelo Ministério da Saúde e Anvisa.
\end{abstract}

Palavras chave: Bioacumulação, peixes, Metais pesados, ICP - OES, Laguna dos Patos.

Evaluation of metals in fish from the region of Pontal of Bar, Patos Lagoon, Pelotas-RS-Brazil

\begin{abstract}
Heavy metals have the ability to accumulate in living tissues throughout the food chain. Fish can become the main form of population transfer to these elements, since they are capable of bioaccumulation and bioconcentration. In order to investigate the bioaccumulation in fish in the Pontal of Bar - Patos Lagoon, in Pelotas, were selected three species abundant in the region, Micropogonias Furnieri (Corvina), Mugil Platanus (Tainha) and Netuma Barba (Bagre). Samples of their muscles were analyzed to verify the contents of $\mathrm{Al}, \mathrm{Cd}, \mathrm{Cr}, \mathrm{Cu}, \mathrm{Fe}, \mathrm{Mn}, \mathrm{Ni}, \mathrm{Pb}$ and $\mathrm{Zn}$. Samples in quintuplicate suffered $\mathrm{HNO}_{3} / \mathrm{HCIO}_{4}$ acid digestion, and the extracts analyzed by inductively coupled plasma - optical emission spectrometry (ICP-OES). The levels of chromium found in all species were above those established by Brazilian legislation (Decree-Law 55.871-65), which determines a maximum level tolerated for chromium $0.1 \mathrm{mg} \mathrm{kg}^{-1}$. The $82.46 \pm 4.69 \% \mathrm{mg} \mathrm{kg}^{-1}$ of zinc in the species, Netuma barba, also exceeded the allowable limit. The cadmium concentrations found in the species Micropogonias furnieri, $0.92 \%$ $\pm 8.16 \% \mathrm{mg} \mathrm{kg}^{-1}$ were close to the limit. The other analytes were found at levels below those established by the Ministry of Health and ANVISA.
\end{abstract}

Keywords: Bioacumulation, fishes, Heavy metals, ICP - OES, Patos Lagoon .

*Corresponding author: Pedro Sanches Filho; e-mail: pjsans@ibest.com.br 


\section{INTRODUÇÃO}

Quase todos os elementos químicos estão envolvidos em ciclos fechados na natureza, em concentrações que não causam efeitos nocivos aos organismos, movendo-se entre os vários compartimentos ambientais em velocidades e extensões variadas (Markert, 1998). Entretanto, um dos aspectos mais graves da introdução de substâncias químicas nesses compartimentos, é a sua bioacumulação na cadeia alimentar existente nos ambientes aquáticos e terrestres (Virga et al., 2007).

Uma vez que o metal se acumula nos tecidos do organismo, esta concentração expressa uma medida integrada do tempo ao qual o animal ficou efetivamente exposto ao elemento (Bryan et al., 1980). Determinar as concentrações dos contaminantes na biota significa monitorar os níveis da fração "biodisponível" destes nos ecossistemas. A toxicidade de metais pesados em organismos aquáticos e seu equilíbrio são influenciados por alguns fatores limnológicos, tais como $\mathrm{pH}$, alcalinidade, dureza, matéria orgânica, sólidos totais e cargas de sedimentação (Aprile et al., 2005).

Nos ambientes aquáticos, mais de $90 \%$ da carga de metais está associada ao material particulado em suspensão e ao sedimento (Zheng et al., 2008). A preocupação associada aos químicos presentes no sedimento decorre do fato de muitas espécies da cadeia trófica passarem a maior parte de seu ciclo de vida neste. Surge assim, uma rota para que esses compostos possam ser consumidos por animais "superiores", incluindo aves e o próprio homem (Adams et al., 1992).

Os estuários e a regiões costeiras são muito utilizados para a disposição de efluentes urbanos e industriais, acarretando a contaminação das águas e da vida marinha por diversos poluentes. Pode-se verificar, mundialmente, aumento nos níveis desses contaminantes e essa constatação tem levado à formulação de estratégias para diminuir o impacto causado nesses ecossistemas que sustentam a maior parte da biodiversidade marinha, os principais recursos pesqueiros e as reservas energéticas mundiais (Maia, 2006). Do ponto de vista da saúde, o grau de contaminação desses ecossistemas pode colocar em risco a saúde das populações ribeirinhas que utilizam essas águas tanto para a pesca quanto para o lazer (Maia, 2006).

A Laguna dos Patos situa-se na planície costeira do Rio Grande do Sul, paralelo à costa na direção nordeste (NE) sudeste (SW), e se conecta com o oceano nos municípios do Rio Grande e São José do Norte (Schwochow \& Zanboni, 2007).

O Saco do Laranjal localiza-se dentro da região estuarina da Laguna dos Patos, e é dividido em diversas praias, entre elas estão: Pontal da Barra, Santo Antônio, Valverde, Balneário dos Prazeres e Colônia Z-3.

O estuário da Laguna dos Patos ocupa uma área de 963,8 $\mathrm{Km}$, apresenta um volume de $1,67 \times 10^{9} \mathrm{~m}^{3}$, sendo um ambiente raso, com profundidade média de $1,74 \mathrm{~m}$ (Schwochow \& Zanboni, 2007). Esse estuário, assim como o Pontal da Barra, local da coleta dos peixes analisados, apresentam grande importância ecológica e econômica para a região, e da qual muitas famílias dependem, já que o sustento destas é proveniente da pesca realizada nestes locais.
A ictiofauna do estuário é constituída de cerca de 110 espécies, sendo que muitas destas permanecem na região por um curto período de tempo, enquanto algumas completam todo o seu ciclo de vida no estuário, onde encontram alimento em abundância e proteção contra a predação (Garcia, 2001). Portanto, muitos peixes jovens utilizam o estuário da Laguna dos Patos como zona de "berçário" (Chao et al., 1985). As zonas rasas do estuário (profundidade $<1,5 \mathrm{~m}$ ) apresentam ictiofauna dominada por pequenos estuarinos residentes, como o peixe-rei Atherinella brasiliensis, e espécies marinhas estuarinas dependentes, como as tainhas do gênero Mugil (Vieira et al., 1998). Esta região é muito importante nas fases iniciais (pós-larvas e juvenis) do ciclo de vida de diversas espécies capturadas pela pesca artesanal, como a Corvina (Micropogonias furnieri) e a tainha (Mugil. platanus) (Vieira \& Scalabrin 1991). O estuário da laguna dos Patos também é de fundamental importância para o Bagre (Netuma barba), já que essa espécie utiliza esse ambiente para reproduzirse e desenvolver-se, na fase juvenil, retornando para o mar quando adultos, sendo por este motivo considerados como migratórios.

A comercialização dessas espécies é feita por muitos pescadores que fazem da pesca seu meio de subsistência. Como estes peixes são vendidos para a população local e também para o Mercado Público Central, que os revende para a população em geral, a análise de metais pesados é importante para avaliar a qualidade do pescado e os possíveis riscos para a saúde humana através do consumo destes.

Com a finalidade de avaliar a biodisponibilidade destes contaminantes através de seus níveis de contaminação nesta importante fonte de proteína para a população em geral, este trabalho tem como objetivo principal a determinação do teor de metais, $\mathrm{Al}, \mathrm{Cd}, \mathrm{Cr}, \mathrm{Cu}, \mathrm{Fe}, \mathrm{Mn}, \mathrm{Ni}, \mathrm{Pb}$ e Zn em espécies de peixes Micropogonias furnieri (Corvina), Mugil platanus (Tainha) $e$ Netuma barba (Bagre), coletados no Pontal da Barra- Saco do Laranjal- Laguna dos Patos, junto ao município de Pelotas.

\section{MATERIAL E MÉTODOS}

\section{Área de estudo}

O Pontal da Barra, identificado na figura 1 localiza-se dentro da região estuarina da Laguna dos Patos, no município de Pelotas exatamente na comunicação com o canal São Gonçalo (região delimitada aproximadamente pelos pontos

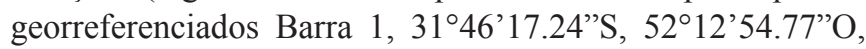

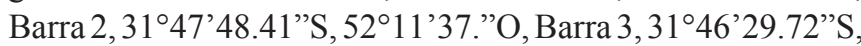
$52^{\circ} 11^{\prime} 11^{\prime \prime} O$ Barra 4, $\left.31^{\circ} 47^{\prime} 20^{\prime \prime} \mathrm{S}, 52^{\circ} 13^{\prime} 28^{\prime \prime} \mathrm{O}\right)$, e, foi escolhida como área de amostragem devido a grande importância ambiental e econômica para a região.

\section{Coleta de amostra}

A coleta dos peixes foi realizada em março de 2011, com o auxílio de pescadores do local. 


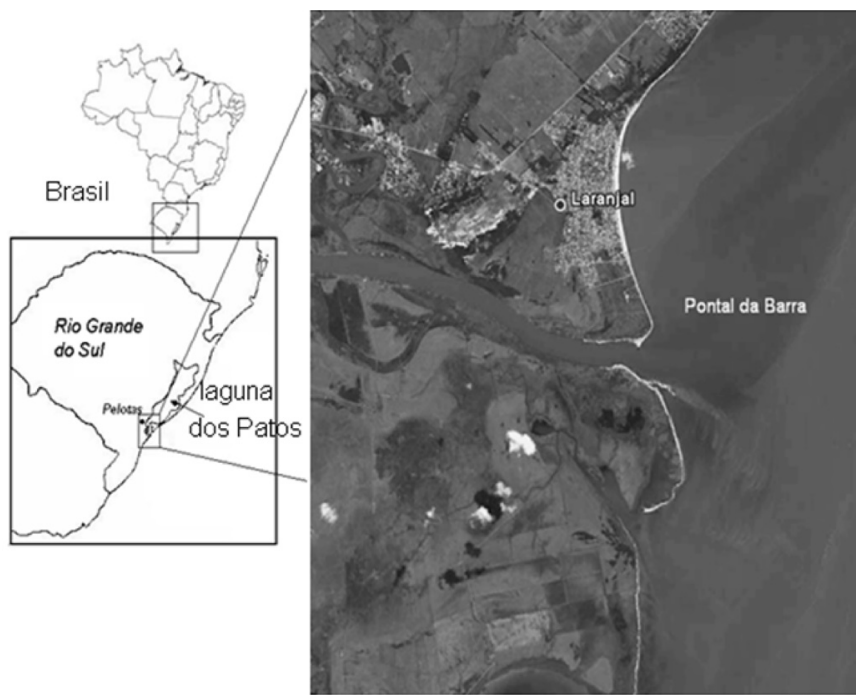

Figura 1 - Localização da área de estudo. Fonte: Google Earth.

Foram capturadas cinco unidades de cada espécie: Micropogonias furnieri (Corvina), Mugil platanus (Tainha) $e$ Netuma barba (Bagre).

Após a coleta, as amostras foram acondicionadas em sacos plásticos e armazenadas a $-20{ }^{\circ} \mathrm{C}$ até dissecação.

\section{Preparação dos materiais para análise}

Toda a vidraria utilizada neste trabalho permaneceu previamente em imersão por $24 \mathrm{~h} \mathrm{em} \mathrm{HNO}_{3}$ a $10 \%$, com a finalidade de evitar a contaminação por metais.

\section{Preparação de amostras para análise}

As amostras foram levadas até a temperatura ambiente e em seguida, dissecadas, sendo utilizado apenas os músculos neste estudo.

Após, as amostras foram secas a $60{ }^{\circ} \mathrm{C}$ em forno estufa para digestão ácida e análise dos metais, e a $105^{\circ} \mathrm{C}$ até peso constante para a determinação da umidade.

\section{Reagentes}

Todos os reagentes utilizados foram de grau analítico. Ácido Nítrico concentrado (65\% p/p) e Ácido Perclórico (70\% p/p) sendo utilizados na digestão das amostras. O Padrão ICP-Multielementos VIII - $100 \mathrm{mg} \mathrm{L}^{-1}$ (Merck) foi utilizado para o preparo das soluções de trabalho. Para as diluições usou-se água Mili-Q.

\section{Estudo dos métodos de digestão}

Para decidir qual metodologia a ser utilizada para a abertura das amostras, primeiramente, foi realizada uma comparação entre dois métodos.

Utilizou-se amostra de Mugil platanus (Tainha).

$\mathrm{O}$ primeiro método estabelece que se pese $1 \mathrm{~g}$ da amostra seca em cadinho de porcelana, previamente calcinado em forno a temperatura de $450^{\circ} \mathrm{C}$ por 1 hora. As amostras são aquecidas a essa mesma temperatura por cerca de 4 horas, até obter um resíduo de cor branca ou cinza claro. O resíduo é dissolvido em $\mathrm{HNO}_{3}(25 \% \mathrm{v} / \mathrm{v})$ e, após, avolumado a $25 \mathrm{~mL}$ com água Mili - Q (Tüzen, 2003).

No segundo método, a mesma quantidade de amostra foi pesada, digerida com uma mistura de $\mathrm{HNO}_{3}$ concentrado e $\mathrm{HClO}_{4}(2: 1 \mathrm{v} / \mathrm{v})$, aquecida a $60^{\circ} \mathrm{C}$ por 72 horas e avolumada da mesma maneira que o método anterior (Dural et al., 2007).

Após a digestão, todas as amostras foram analisadas por Espectrometria de emissão óptica com plasma acoplado indutivamente (ICP-OES), e a partir dos resultados decidiu-se adotar o segundo método de digestão.

As análises foram realizadas em quintuplicatas. A mistura de Ácido Nítrico e Perclórico foi utilizada como branco para avaliar o potencial de contaminação das amostras em toda a digestão.

\section{Determinação de metais}

Após a digestão, a determinação quantitativa dos metais foi realizada por ICP-OES, com equipamento da marca Perkin Elmer, modelo Optima 3300, utilizando plasma de argônio com vazão de $15 \mathrm{~L} \mathrm{~min}^{-1}$, e potência de radiofrequência de 1.300 W. As leituras das amostras foram feitas em quintuplicatas.

As curvas analíticas foram realizadas com soluções diluídas, nas concentrações $0,1,0,5,1,0,5,0$ e $10,0 \mathrm{mg} \mathrm{L}^{-1}$, a partir do Padrão ICP para multielementos $100 \mathrm{mg} \mathrm{L}^{-1}$ (Merck), obtendo-se os coeficientes de correlação, angular e linear. O padrão $1,0 \mathrm{mg} \mathrm{L}^{-1}$ foi analisado 11 vezes para avaliar a precisão. Para avaliar a recuperação do método foi analisada amostra certificada DORM 3 (Canadá)

A leitura do branco foi realizada 10 vezes para calcular o limite de quantificação (LQ) e o limite de detecção (LD) do equipamento, que foram calculados considerando o sinal médio do branco mais 10 vezes o desvio padrão (SD) para o LQ e o sinal médio do branco mais 3 vezes o SD para o LD, conforme IUPAC, 1997.

\section{RESULTADOS E DISCUSSÃO}

Conforme a tabela 1 pode-se observar que o método II apresentou melhores resultados de recuperação. O processo de calcinação pode levar a perdas, principalmente do $\mathrm{Pb}, \mathrm{Cu}$, $\mathrm{Cr}$ e $\mathrm{Fe}$ o que justifica os menores resultados para a maioria dos analitos. Também os valores superiores de desvio padrão relativo expressos em \% (RSD) indicam uma menor precisão no método.

A tabela 2 mostra os coeficientes de correlação $\left(\mathrm{R}^{2}\right)$, angular (a) e linear (b) para os metais analisados, obtidos através da curva de calibração; LQ e LD do equipamento e, também, desvio padrão relativo (RSD). Podemos observar a resposta linear para faixa de trabalho com os $\mathrm{r}^{2} 0,999$, os limites de detecção localizaram-se entre 0,018 para $\mathrm{Mn}$ a $0,273 \mathrm{mg} \mathrm{L}^{-1}$ para o Zn e os LQ localizaram-se entre 0,019 para Mn a 0,296 
Tabela 1- Resultados obtidos em peso seco comparando o método I utilizado por Tüzen, M., 2003 e o método II utilizado por Dural et al., 2007 para a amostra de Tainha.

\begin{tabular}{lcc}
\hline Elemento & Método I $\left(\mathrm{mg} \mathrm{Kg}^{-1}\right)$ & Método II $\left(\mathrm{mg} \mathrm{Kg}^{-1}\right)$ \\
\hline $\mathrm{Al}$ & $10,68 \pm 15,66 \%$ & $10,35 \pm 11,94 \%$ \\
$\mathrm{Cd}$ & $0,55 \pm 17,90 \%$ & $0,83 \pm 10,75 \%$ \\
$\mathrm{Cr}$ & $0,65 \pm 5,03 \%$ & $1,08 \pm 4,14 \%$ \\
$\mathrm{Cu}$ & $1,43 \pm 23,01 \%$ & $5,64 \pm 8,11 \%$ \\
$\mathrm{Fe}$ & $15,00 \pm 17,21 \%$ & $22,48 \pm 15,62 \%$ \\
$\mathrm{Mn}$ & $1,00 \pm 15,53 \%$ & $0,90 \pm 10,58 \%$ \\
$\mathrm{Ni}$ & $0,44 \pm 13,60 \%$ & $0,41 \pm 9,18 \%$ \\
$\mathrm{~Pb}$ & $\mathrm{ND}$ & $1,13 \pm 10,18 \%$ \\
$\mathrm{Zn}$ & $26,60 \pm 9,20 \%$ & $26,40 \pm 7,88 \%$ \\
\hline não detectado & &
\end{tabular}

$m g L^{-1}$ para o $\mathrm{Zn}$, refletindo a excelente performance do aparelho gerando pequenos desvios padrão para o sinal do branco, o que é confirmado pelo RSD menor que $2 \%$ para maioria dos analitos. Os Valores de recuperação obtidos a partir da amostra certificada DORM 3 variaram entre $86,8 \%$ para o Níquel a $98,7 \%$ para o chumbo apresentados também na tabela 2.

Os níveis de $\mathrm{Al}, \mathrm{Cd}, \mathrm{Cr}, \mathrm{Cu}, \mathrm{Fe}, \mathrm{Mn}, \mathrm{Ni}, \mathrm{Pb}$ e $\mathrm{Zn}$ nos músculos dos peixes Micropogonias furnieri (Corvina), Mugil platanus (Tainha) e Netuma barba (Bagre), assim como o LD e LQ do método são mostrados na tabela 3.

Para fins de comparação com a legislação, as concentrações de metais foram convertidas de peso seco (p.s) para peso úmido (p.u), sendo que para isso as médias das concentrações

Tabela 2- Coeficientes de correlação $\left(\mathrm{R}^{2}\right)$, angular (a) e linear (b), obtidos através da curva de calibração, LQ e LD do equipamento, RSD

\begin{tabular}{|c|c|c|c|c|c|c|c|c|c|}
\hline $\begin{array}{l}\text { Ele- } \\
\text { mento }\end{array}$ & $\lambda(\mathrm{nm})$ & a & $\mathrm{b}$ & $\mathrm{R}^{2}$ & $\begin{array}{c}\mathrm{LD} \\
\mathrm{mg} \mathrm{L}^{-1}\end{array}$ & $\begin{array}{c}\mathrm{LQ} \\
\mathrm{mg} \mathrm{L}^{-1}\end{array}$ & $\begin{array}{l}\text { RSD } \\
(\%)\end{array}$ & $\begin{array}{c}\text { DORM } 3 \\
\mathrm{mg} \mathrm{kg}^{-1}\end{array}$ & $\begin{array}{c}\text { Rec } \\
\%\end{array}$ \\
\hline $\mathrm{Al}$ & 396,153 & 4175 & $-262,7$ & 0,999 & 0,211 & 0,277 & 1,87 & $\mathrm{x}$ & $\mathrm{x}$ \\
\hline $\mathrm{Cd}$ & 228,802 & 5177 & $-115,2$ & 0,999 & 0,027 & 0,035 & 1,31 & $0,29 \pm 0,02$ & 87,5 \\
\hline $\mathrm{Cr}$ & 267,716 & 17180 & $-394,4$ & 0,999 & 0,065 & 0,076 & 1,33 & $1,89 \pm 0,17$ & 89,4 \\
\hline $\mathrm{Cu}$ & 327,393 & 19034 & $-85,70$ & 0,999 & 0,191 & 0,212 & 1,50 & $15,5 \pm 0,63$ & 93,2 \\
\hline $\mathrm{Fe}$ & 238,204 & 6591 & $-249,5$ & 0,999 & 0,234 & 0,256 & 1,65 & $347 \pm 2,00$ & 90,8 \\
\hline $\mathrm{Mn}$ & 257,610 & 90396 & -1136 & 0,999 & 0,018 & 0,019 & 1,57 & $\mathrm{x}$ & $\mathrm{x}$ \\
\hline $\mathrm{Ni}$ & 231,604 & 6939 & $-24,60$ & 0,999 & 0,033 & 0,047 & 1,02 & $1,28 \pm 0,24$ & 86,8 \\
\hline $\mathrm{Pb}$ & 220,353 & 969,1 & $-8,443$ & 0,999 & 0,060 & 0,106 & 4,170 & $0,395 \pm 0,05$ & 98,7 \\
\hline $\mathrm{Zn}$ & 206,200 & 4355 & $-33,53$ & 0,999 & 0,273 & 0,296 & 0,765 & $51,30 \pm 3,10$ & 97,3 \\
\hline \multicolumn{10}{|c|}{$\lambda=$ Comprimento de onda da linha de emissão } \\
\hline \multicolumn{10}{|c|}{$\mathrm{LQ}=$ Limite de quantificação } \\
\hline \multicolumn{10}{|c|}{$\mathrm{LD}=$ Limite de detecção } \\
\hline \multicolumn{10}{|c|}{$\mathrm{RSD}=$ Desvio padrão relativo } \\
\hline & . & & & & & & & & \\
\hline
\end{tabular}

$\mathrm{Rec}=$ recuperação dos analitos a partir do material de referencia expressa em porcentagem.

$\mathrm{x}=$ não descrito no material de referencia
Tabela 3- Concentração de metais em músculos de três espécies de peixes, em $\mathrm{mg} \mathrm{Kg}^{-1}$ de peso seco \pm desvio padrão relativo expresso em porcentagem referentes as quintuplicatas de digestão, LD e LQ do método, em mg Kg-1.

\begin{tabular}{lccccc}
\hline Elemento & Bagre & Corvina & Tainha & LD & LQ \\
\hline $\mathrm{Al}$ & $9,01 \pm 2,79 \%$ & $10,41 \pm 7,21 \%$ & $10,35 \pm 11,94 \%$ & 5,27 & 6,92 \\
$\mathrm{Cd}$ & $0,66 \pm 3,14 \%$ & $1,17 \pm 8,16 \%$ & $0,83 \pm 10,75 \%$ & 0,67 & 0,87 \\
$\mathrm{Cr}$ & $1,06 \pm 7,75 \%$ & $1,06 \pm 7,69 \%$ & $1,08 \pm 4,14 \%$ & 1,62 & 1,90 \\
$\mathrm{Cu}$ & $5,36 \pm 6,55 \%$ & $5,16 \pm 3,78 \%$ & $5,64 \pm 8,11 \%$ & 4,77 & 5,30 \\
$\mathrm{Fe}$ & $21,48 \pm 11,72 \%$ & $15,41 \pm 7,40 \%$ & $22,48 \pm 15,62 \%$ & 5,85 & 6,40 \\
$\mathrm{Mn}$ & $0,93 \pm 6,98 \%$ & $0,89 \pm 10,18 \%$ & $0,90 \pm 10,58 \%$ & 0,45 & 0,47 \\
$\mathrm{Ni}$ & $0,62 \pm 6,41 \%$ & $0,51 \pm 14,49 \%$ & $0,41 \pm 9,18 \%$ & 0,82 & 1,17 \\
$\mathrm{~Pb}$ & $1,11 \pm 6,33 \%$ & $0,58 \pm 13,75 \%$ & $1,13 \pm 10,18 \%$ & 1,50 & 2,65 \\
$\mathrm{Zn}$ & $123,07 \pm 4,69 \%$ & $26,54 \pm 3,15 \%$ & $26,40 \pm 7,88 \%$ & 6,82 & 7,40 \\
\hline $\mathrm{LD}=$ Limite de detecção & & & & \\
$\mathrm{LQ}=$ & & & &
\end{tabular}

dos metais encontrados na corvina, na tainha e no bagre foram multiplicadas respectivamente por 0,$79 ; 0,72$ e 0,67 ; já que o fator de conversão utilizado para se obterem os valores para peso úmido foi a média da porcentagem de umidade das espécies de peixes, que apontaram respectivamente $21 \%$ (Micropogonias furnieri), 28\% (Mugil platanus) e 33\% (Netuma barba) de umidade. Os valores encontrados estão mostrados na tabela 4 .

Podemos observar que todos os metais estudados foram detectados nas três espécies. A concentração de cromo apresentou-se acima do limite máximo tolerável (LTM) estabelecido pela legislação brasileira (Decreto Lei 55.8711965), 0,1 $\mathrm{mg} \mathrm{Kg}^{-1}$, nas três espécies estudadas, Micropogonias furnieri, Mugil platanus e Netuma barba, assim como a de zinco na espécie Netuma barba, que também mostrou-se acima do permitido pela mesma legislação, $50 \mathrm{mg} \mathrm{Kg}^{-1}$. A concentração de cádmio na espécie Micropogonias furnieri apresentou-se próxima do limite máximo tolerável (LTM), $1 \mathrm{mg} \mathrm{Kg}^{-1}$, sendo que todos os outros metais analisados apresentaram-se dentro dos limites permitidos por lei para o consumo humano.

Tabela 4- Concentração de metais em peso úmido e limite máximo de tolerância brasileiro, em $\mathrm{mg} \mathrm{Kg}^{-1}$.

\begin{tabular}{lcccc}
\hline $\begin{array}{l}\text { Elemen- } \\
\text { to }\end{array}$ & $\begin{array}{c}\text { LTM da } \\
\text { Legislação } \\
\text { Brasileira em } \\
\mathrm{mg} \mathrm{Kg}^{-1}\end{array}$ & \multicolumn{2}{c}{ Concentração em mg Kg-1 em peso úmido } \\
\hline $\mathrm{Al}$ & ---- & $6,04 \pm 2,79 \%$ & $8,22 \pm 7,21 \%$ & $7,45 \pm 11,94 \%$ \\
$\mathrm{Cd}$ & 1,00 & $0,44 \pm 3,14 \%$ & $0,92 \pm 8,16 \%$ & $0,59 \pm 10,75 \%$ \\
$\mathrm{Cr}$ & 0,10 & $0,71 \pm 7,75 \%$ & $0,84 \pm 7,69 \%$ & $0,78 \pm 4,14 \%$ \\
$\mathrm{Cu}$ & 30,00 & $3,59 \pm 6,55 \%$ & $4,08 \pm 3,78 \%$ & $4,06 \pm 8,11 \%$ \\
$\mathrm{Fe}$ & ---- & $14,39 \pm 11,72 \%$ & $12,17 \pm 7,40 \%$ & $16,18 \pm 15,62 \%$ \\
$\mathrm{Mn}$ & ---- & $0,62 \pm 6,98 \%$ & $0,70 \pm 10,18 \%$ & $0,65 \pm 10,58 \%$ \\
$\mathrm{Ni}$ & 5,00 & $0,41 \pm 6,41 \%$ & $0,40 \pm 14,49 \%$ & $0,29 \pm 9,18 \%$ \\
$\mathrm{~Pb}$ & 2,00 & $0,74 \pm 6,33 \%$ & $0,46 \pm 13,75 \%$ & $0,81 \pm 10,18 \%$ \\
$\mathrm{Zn}$ & 50,00 & $82,46 \pm 4,69 \%$ & $20,97 \pm 3,15 \%$ & $19,01 \pm 7,88 \%$ \\
\hline
\end{tabular}

LTM = Limite máximo tolerável 
A bioacumulação de metais pesados em peixe é dependente da espécie. Hábitos alimentares e estilo de vida das espécies estão fortemente relacionadas ao nível de acumulação (Yusal et al., 2008). O acúmulo de metais pesados no organismo é resultado da diferença entre a absorção e depuração e isso é o fator mais importante na acumulação de metal (Canli, 2003). A bioacumulação é definida como a transferência para os organismos de determinada quantidade de elementos químicos ou substâncias que se encontram no ambiente (Cohen, 1985). Os processos de biodisponibilidade dependerão do tipo de absorção realizada pelo organismo, do tamanho das partículas ou da fase em que os metais se encontram (dissolvidos ou particulado) (Jesus et al., 2008).

A poluição por metais pesados está relacionada a metais presentes na forma dissolvida ou à matéria particulada em suspensão na coluna d'água (Santos, 2006).

Em ecossistemas aquáticos peixes são considerados como bons indicadores da saúde global do sistema devido à sua relativa elevada posição na cadeia alimentar (Adam et al., 1993).

Os peixes analisados neste trabalho são considerados espécies bentônicas e possuem diferentes hábitos alimentares, sendo que a tainha (Mugil platanus) é um peixe planctívoro; o bagre (Netuma barba) - peixe onívoro e corvina (Micropogonias furnieri), peixe carnívoro. Baseado nessa classificação sugere-se que a concentração de $\mathrm{Cd}$ tenha sido maior na corvina, quando comparado as outras espécies, devido ao fato dessa espécie possuir hábito alimentar carnívoro, o que indica que o acúmulo de metais pode ser maior pela razão da biomagnificação ao longo da cadeia trófica, já que esse peixe se alimenta de indivíduos de níveis tróficos maiores, os quais por estarem em uma posição mais elevada na cadeia alimentar apresentam-se mais contaminados. O alto teor de zinco encontrado no bagre também pode estar relacionado ao seu hábito alimentar, já que esta espécie por ser onívora alimentase tanto de vegetais quanto animais, tendo preferência por invertebrados aquáticos como larvas, insetos e detritos de fundo. Os elevados níveis de cromo encontrados nas espécies Netuma barba, Micropogonias furnieri e Mugil platanus provavelmente devem-se aos mesmos motivos citados anteriormente, hábito alimentar, porém como a espécie Mugil platanus se alimenta apenas de plantas, sendo essa o menor nível trófico na cadeia, e, portanto o que deveria estar menos contaminado, isso indica, então, a possível contaminação em vários compartimentos desse local do estudo.

O conhecimento de concentrações de metais pesados em peixes é importante tanto com relação à gestão da natureza e saúde humana (Dural et al., 2007).

Para os humanos, a incorporação de metais pode variar de acordo com o tipo étnico, idade, sexo, local de residência (área urbana ou rural, poluída ou não), número de fontes de contaminação ao qual a população está exposta (ar, água, alimento, solo, utensílio, cigarro, etc.), quantidade de contaminantes que é ingerida de cada fonte, etc. (Virga et al., 2007).
Levando-se em conta todos estes fatos, algumas agências de saúde têm proposto limites máximos provisórios de ingestão diária de metais para a população como uma forma de orientação de vigilância sanitária. Os valores provisórios de ingestão diária tolerável (PTDI), sugeridos pela Organização Mundial de Saúde para os metais são apresentados na tabela 5. Estes valores foram determinados considerando uma massa média para o corpo humano de $60 \mathrm{Kg}$ (Virga et al., 2007).

Utilizando-se os resultados descritos na tabela 4, determinou-se a ingestão média de metal pela população, por $100 \mathrm{~g}$ (peso úmido) de carne das espécies dos peixes em estudo, e os valores obtidos estão mostrados na tabela 5. Assim, observa-se a contribuição significativa ao PTDI em virtude do consumo de pescado das três espécies principalmente em relação aos metais $\mathrm{Cd}$ e $\mathrm{Pb}$, com especial atenção ao cádmio, já que as contribuições desse metal em função da ingestão do bagre, corvina e tainha são respectivamente $73 \%, 153,3 \%$ e $98 \%$ do total recomendado para ingestão diária, o que significa dizer que ao consumir $100 \mathrm{~g} /$ dia de bagre, corvina e tainha, está ingerindo-se, respectivamente; $0,044 \mathrm{mg}, 0,092 \mathrm{mg}$ e $0,059 \mathrm{mg}$ de cádmio. Sendo, então, que ao consumir apenas $100 \mathrm{~g} \mathrm{dia}^{-1}$ de corvina a quantidade de ingestão diária tolerável para o Cd é ultrapassada, tendo-se, assim, a acumulação deste metal no organismo.

Cabe ressaltar que o nível de ingestão deve depender dos hábitos da população/indivíduo que irá consumi-los. Por exemplo, se o individuo é fumante, o nível de cádmio que ele possui no sangue é maior do que um não fumante e, portanto, o seu limite de ingestão deveria ser menor. Muitos outros fatores podem influenciar esse limite como a qualidade do ar, da água, do solo, etc (Virga et al., 2007).

Como todos os peixes apresentaram valores de $\mathrm{Cr}$ acima do permitido pela legislação brasileira, pode-se dizer que estão impróprios para consumo humano, uma vez que seu consumo pode ocasionar doenças relativas a ingestão desse metal, sendo que as espécies Netuma barba e Micropogonias furnieri são ainda mais críticas devido ao fato de também apresentarem respectivamente, $\mathrm{Zn}$ acima do limite legal e Cd muito próximo do máximo estabelecido por lei, o que

Tabela 5- Contribuição do consumo de pescado pela população, em relação ao valor provisório de ingestão diária tolerável (PTDI), sugerido pela Organização Mundial de Saúde (OMS), para uma pessoa adulta de $60 \mathrm{Kg}$.

\begin{tabular}{|c|c|c|c|c|}
\hline \multirow{2}{*}{ Metal } & \multirow{2}{*}{ PTDI OMS } & \multicolumn{3}{|c|}{ Ingestão média. $100 \mathrm{~g}^{-1}$ (peso úmido) de pescado } \\
\hline & & Bagre & Corvina & Tainha \\
\hline $\mathrm{Cd}$ & $0,06 \mathrm{mg} \mathrm{dia}{ }^{-1}$ & $\begin{array}{c}0,044 \mathrm{mg} \mathrm{dia}{ }^{-1} \\
(73,0 \%)\end{array}$ & $\begin{array}{c}0,092 \mathrm{mg} \mathrm{dia}{ }^{-1} \\
(153,3 \%)\end{array}$ & 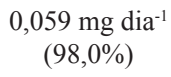 \\
\hline $\mathrm{Cr}$ & $1,0 \mathrm{mg} \mathrm{dia}^{-1}$ & $\begin{array}{c}0,071 \mathrm{mg} \mathrm{dia}{ }^{-1} \\
(7,1 \%)\end{array}$ & $\begin{array}{c}0,084 \mathrm{mg} \mathrm{dia}{ }^{-1} \\
(8,4 \%)\end{array}$ & $\begin{array}{c}0,078 \mathrm{mg} \mathrm{\textrm {dia } ^ { - 1 }} \\
(7,8 \%)\end{array}$ \\
\hline $\mathrm{Cu}$ & $3,0 \mathrm{mg} \mathrm{dia}^{-1}$ & $\begin{array}{c}0,359 \mathrm{mg} \mathrm{dia}^{-1} \\
(12,0 \%)\end{array}$ & $\begin{array}{c}0,408 \mathrm{mg} \mathrm{dia}^{-1} \\
(14,0 \%)\end{array}$ & $\begin{array}{c}0,406 \mathrm{mg} \mathrm{dia}{ }^{-1} \\
(14,0 \%)\end{array}$ \\
\hline $\mathrm{Ni}$ & $0,30 \mathrm{mg} \mathrm{dia}^{-1}$ & $\begin{array}{c}0,041 \mathrm{mg} \mathrm{dia}{ }^{-1} \\
(14,0 \%)\end{array}$ & $\begin{array}{c}0,040 \mathrm{mg} \mathrm{dia}{ }^{-1} \\
(13,0 \%)\end{array}$ & $\begin{array}{l}0,029 \mathrm{mg} \mathrm{\textrm {dia } ^ { - 1 }} \\
\quad(10,0 \%)\end{array}$ \\
\hline $\mathrm{Pb}$ & $0,21 \mathrm{mg} \mathrm{dia}^{-1}$ & $\begin{array}{c}0,074 \mathrm{mg} \mathrm{dia}{ }^{-1} \\
(35,0 \%)\end{array}$ & $\begin{array}{c}0,046 \mathrm{mg} \mathrm{dia}{ }^{-1} \\
(22,0 \%)\end{array}$ & 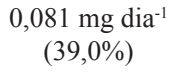 \\
\hline $\mathrm{Zn}$ & $60,0 \mathrm{mg} \mathrm{dia}{ }^{-1}$ & $\begin{array}{c}8,246 \mathrm{mg} \mathrm{dia}^{-1} \\
(14 \%)\end{array}$ & $\begin{array}{c}2,097 \mathrm{mg} \mathrm{dia}^{-1} \\
(3,5 \%)\end{array}$ & $\begin{array}{c}1,901 \mathrm{mg} \mathrm{dia}{ }^{-1} \\
(3,2 \%)\end{array}$ \\
\hline
\end{tabular}


pode aumentar a possibilidade de intoxicação alimentar pelo consumo dessas espécies.

A ingestão de concentrações elevadas de cromo hexavalente pode produzir mal estar estomacal, úlceras, convulsões, danos ao fígado e rins, e até a morte, também pode ser considerado carcinogênico aos seres humanos (USEPA, 1984b).

Apesar do zinco ser considerado um elemento essencial para a fisiologia de organismos, o envenenamento de seres humanos pode ocorrer, resultando em doenças pulmonares, gastroenterite, febre, vômitos, problemas de coordenação muscular e desidratação. Nos peixes ele pode obstruir os espaços interlamelares, bloqueando o movimento de respiração, além de promover o atraso do crescimento e maturação ( Lima et al., 2002). No estudo de Marcovecchio (2004), no estuário de La Plata Argentina, encontrou 0,34 $\pm 0,05$ e 48,8 $\pm 3,6 \mathrm{mg} \mathrm{Kg}^{-1}$ de Cd e $\mathrm{Zn}$, respectivamente em amostra de músculo de Mugil platanus, já no Micropogonias furnieri não foi detectado $\mathrm{Cd}$, sendo o $\mathrm{Zn}$ encontrado em concentrações de 20,5 4,6 mg $\mathrm{Kg}^{-1}$ tais valores assemelham-se aos deste trabalho.

Nos seres humanos, prolongada ingestão excessiva de zinco podem conduzir a deficiência em ferro e cobre, náuseas, vômitos, febre, dor de cabeça, cansaço e dor abdominal. Não há nenhum relatório de possíveis carcinogenicidade de zinco e compostos em seres humanos (Fosmire, 1990).

As implicações de saúde da exposição de cádmio são agravadas pela incapacidade relativa dos seres humanos de excretar cádmio (Dural et al., 2007). A intoxicação por cádmio afeta principalmente os rins, e os efeitos provocados por sua ingestão são distúrbios gastrointestinais.

\section{CONCLUSÃO}

Foi encontrado Cr, em nível acima do limite legal, nas três espécies estudadas, Netuma barba, Micropogonias furnieri e Mugil platanus, assim como Zn, também em concentrações acima do permitido por lei, na espécie Netuma barba. Na espécie Micropogonias furnieri encontrou-se Cd próximo ao valor máximo estipulado

Acredita-se que a origem desses elementos esteja relacionada a resíduos urbanos e industriais, e, também, a atividade agropecuária.

Como o resultado obtido para cromo refere-se ao cromo total, sugere-se estudo de especiação desse elemento, a fim de saber em quais formas está presente no local de estudo.

Devido ao potencial de toxicidade desses elementos traço, cujas concentrações mostraram-se elevadas nos peixes, sugerem-se estudos futuros para o contínuo monitoramento da biota da Laguna dos Patos, mais especificamente do Pontal da Barra, já que esta região é de fundamental importância ecológica, econômica e social, e também pelo fato de que os peixes deste local servem tanto como fonte de renda quanto para consumo próprio pela população local. Os valores também refletem a biodisponibilidade de cada um dos metais.

\section{AGRADECIMENTOS}

Agradecemos ao grupo de trabalho de pesquisa do laboratório de contaminantes ambientais, LACA, ao Curso de Química, Tecnólogos em Gestão, Saneamento Ambiental e Pró-Reitoria de Pesquisa, Inovação e Pós-Graduação do Instituto Federal de Educação Ciência e Tecnologia Sul-riograndense, campus Pelotas pela colaboração de essencial importância neste trabalho.

\section{REFERÊNCIAS}

ADAM, S. M., BROWN, A. M. \& GOEDE, R. W., 1993, A quantitative health assessment index for rapid evaluation of fish condition in the field. Transactions of the American Fisheries Society, 122: 63-73. http://dx.doi.org/10.1577/1548-8659 (1993) $122<0063$ :AQHAIF>2.3.CO;2

ADAMS, W. J., KIMERLE, R. A. \& BARNETT JR, J. W., 1992, Sediment quality and aquatic life assessment. Env Sci Tech., 26(10): 1864-1875. http://dx.doi.org/10.1021/es00034a001

BRYAN, G. W., LANGSTON, W. J. \& HUMMERTONE, L. G., 1980, The use of biological indicator of heavy metal contamination in estuaries: with special reference to an assessment of biological availability of metals in estuarine sediments from south-west Britain. Occ. Publ. Mar. Biol. Ass., UK. 1: 1-73.

CANLI, M. \& ATLI, G., 2003, The relationships between heavy metal $(\mathrm{Cd}, \mathrm{Cr}, \mathrm{Cu}, \mathrm{Fe}, \mathrm{Pb}, \mathrm{Zn}$ ) levels and the size of six Mediterranean fish species. Environ. Pollut., 121: 129-136. http://dx.doi. org/10.1016/S0269-7491(02)00194-X

CHAO, L. H., PEREIRA, L. E. \& VIEIRA, J. P., 1985, Estuarine fish community of the dos Patos Lagoon, Brazil. A baseline study. In: A. Yanez-Arancibia (Ed.). Fish Community Ecology in Estuarines and Coastal Lagoons: Towards an Ecosystem Integration, 429-450p.

COHEN, B. L., 1985, Bioaccumulation factor in marine organism. Health Physics. 49(6): 1290-1294.

DECRETO LEI n ${ }^{\circ}$ 55.781, de 26 de março de 1965: "Princípios Gerais para o Estabelecimento de Níveis Máximos de Contaminantes Químicos em Alimentos" e seu anexo "Limites máximos de tolerância para contaminantes inorgânicos". Disponível em: $<$ www.anvisa.gov.br/legis/decretos/55871_65.htm>. Acesso em: junho 2011.

DURAL, M., GÖKSU, M. Z. L. \& ÖZAK, A. A., 2007, Investigation of heavy metal levels in economically important fish species captured from the Tuzla lagoon. Food Chem., 102: 415-421. http://dx.doi.org/10.1016/j.foodchem.2006.03.001.

FOSMIRE, G. J., 1990, Zinc toxicity. Am. J. Clin. Nutr., 51: $225-$ 227.

GARCIA, A. M. \& VIEIRA, J. P., 2001, O aumento da diversidade de peixes no estuário da Lagoa dos Patos durante o episódio El Nino 1997-1998. Atlântica. 23: 133-152.

IUPAC, International Union of Pure and Applied Chemistry, 1997, Chemistry Compendium of Chemical Terminology 2nd Edition.

LIMA, R. G. S., 2002, Evaluation of Heavy Metals in Fish of the Sepetiba and Ilha Grande Bay, Rio de Janeiro, Brazil. Environ. Res., Section A. 89: 171-179. http://dx.doi.org/10.1006/ enrs.2002.4341

JESUS, T. B., FERNANDEZ, L. G. \& QUEIROZ, A. F. DE S., 2008, Avaliação da concentração de cádmio, cobre, ferro, manganês, níquel e zinco em Anomalocardia brasiliana (Gmelim, 1791) 
provenientes de zonas de manguezal da região de São Francisco do Conde e Madre de Deus, Recôncavo Baiano, BA. J. Braz. Soc. Ecotoxicol., 3(1): 77-84.

MAIA, C. B., ALMEIDA, A. C. M. \& MOREIRA, F. R., 2006, Avaliação do teor de chumbo em Mexilhões da espécie Perna perna na região metropolitana da cidade do Rio de Janeiro. $J$. Braz. Soc. Ecotoxicol., 1(2): 195-198.

MARKERT, B., 1998, Distribution and Biogeochemistry of Inorganic Chemicals in the Environment. In: Schüürmann, G. and Markert, B. (eds.). Ecotoxicology. John Wiley and Sons. Inc and Spekrum Akademischer Verlag, 165-199 p.

MARCOVECCHIO, J. E., The use of Micropogonias furnieri and Mugil liza as bioindicators Of heavy metals pollution in La Plata river estuary, Argentina. Sci. Total Environ., 323(2004) 219-226. http://dx.doi.org/10.1016/j.scitotenv.2003.09.029

SANTOS, D. M., BOSSINI, J. AT., PREUSSLER, K. H., VASCONSELOS, E. C., CARVALHO-NETO, F. S. \& CARVALHO-FILHO, M. A. S., 2006, Avaliação de metais pesados na Baía de Paranaguá, PR, Brasil, sob influência das atividades antrópicas. J. Braz. Soc. Ecotoxicol., 1(2): 157-160.

SCHWOCHOW, R. Q. \& ZANBONI, A. J., 2007, O Estuário da Lagoa dos Patos: um exemplo para o ensino de ecologia no nível médio. Cadernos de Ecologia Aquática. 2 (2): 13-27.

TÜZEN, M., 2003, Determination of heavy metals in fish samples of the middle Black Sea (Turkey) by graphite furnace atomic absorption spectrometry. Food Chem., 80: 119-123. http://dx.doi. org/10.1016/S0308-8146(02)00264-9
USEPA, U. S. ENVIRONMENTAL PROTECTION AGENCY, 1984 Health effects assessment for hexavalent chromium. Report to Office Emergency and Remedial Response, Environmental Criteria and Assessment Office, Cincinnati $(\mathrm{OH})$.

UYSAL, K., EMRE, Y. \& KÖSE, E., 2008, The determination of heavy metal accumulation ratios in muscle, skin and gills of some migratory fish species by inductively coupled plasmaoptical emission spectrometry (ICP-OES) in Beymelek Lagoon (Antalya/Turkey). Microchem. J., 90: 67-70 http://dx.doi. org.:10.1016/j.microc.2008.03.005

VIEIRA, J. P. \& SCALABRIN, C., 1991, Migração reprodutiva da "tainha" (Mugil platanus Gunter, 1980) no sul do Brasil. Atlântica, Rio Grande. 13(1): 131-141.

VIEIRA, J. P., CASTELLO, J. P. \& LE, P., 1998, O ambiente e a biota do estuário da Lagoa dos Patos - Ictiofauna. In: Seeliger, U., Odebrecht, C. \& Castello, J. P. (Eds). Os ecossistemas costeiro e marinho do extremo sul do Brasil. Ecoscientia, Rio Grande. 60-67.

VIRGA, R. H. P., GERALDO, L. P. \& SANTOS, F. H., 2007, Avaliação de contaminação por metais pesados em amostras de siris azuis. Ciênc. Tecnol. Aliment., 27(4): 779-785.

ZHENG, N., WANG, Q., LIANG, Z. \& ZHENG, D., 2008, Characterization of heavy metal concentrations in the sediments of three freshwater rivers in Huludao City, Northeast China. Environ. Pollut., 154: 135-142. http://dx.doi.org /10.1016/j. envpol.2008.01.001 